\title{
Angular and Energy Dependence of Proton Upset in Optocouplers ${ }^{\dagger}$ \\ A. H. Johnston, T. Miyahira, G. M. Swift, S. Guertin, and L. D. Edmonds \\ Jet Propulsion Laboratory \\ Pasadena, California
}

\section{Introduction}

Proton upset effects in optocouplers were reported by LaBel, et al. that showed an unexpected increase in cross section for incident angles above 80 degrees [1]. Although it appeared that the angular dependence was related to direct ionization from protons, the angular dependence was weaker than expected from basic geometrical arguments using a shallow charge collection depth. Later work showed that the angular dependence of proton upset observed in the earlier studies at a single energy could be explained by considering the distribution of proton recoil energies along with the assumption of a deeper charge collection depth, which was consistent with upset tests from heavy ions [2]. However, an experimental test of the underlying assumptions in the latter work has yet to be done.

Protons in space not only arrive over a wide range of incident angles, but also involve a distribution of proton energies. It is necessary to understand both the angular dependence and the dependence of proton upset on energy in order to determine how optocouplers will respond in space. If the angular dependence only occurs for extreme angles of incidence, it will have little impact on the overall cross section because of the narrow acceptance angle. The present work examines mechanisms for proton upset in optocouplers in more detail, investigating the energy dependence and the effects of different load conditions. A model for proton upset is developed, along with a laboratory screening method to determine whether direct ionization is significant for specific device types.

\section{Device Construction and Operation}

Type 6N134 optocouplers from two different manufacturers, Hewlett-Packard and Micropac, were used in this study. Both manufacturers use a sandwich construction, placing a ceramic assembly that contains the light-emitting diode directly over a silicon photodiode. The photodiode is part of an integrated circuit that contains a high-gain amplifier. The diameter of the circular photodiode is nominally $430 \mu \mathrm{m}$ for both types. The physical construction of parts from the two manufacturers is very similar, but the Micropac devices have much thicker die, raising the level of the active device area relative to the bottom of the cavity of the ceramic package.

†The work described in this paper was carried out by the Jet Propulsion Laboratory. California Institute of Technology, under contract with the National Aeronautics and Space Administration, Code AE. Work funded by the NASA Microelectronics Space Radiation Effects Program (MSREP)
The minimum optical power required to switch these devices is weakly dependent on load conditions. Figure 1 shows the transfer characteristics of a typical $6 \mathrm{~N} 134$ optocoupler -- input LED current vs. output voltage with a resistive load -- for three different load conditions. Assuming that LED output power is proportional to load current, approximately $30 \%$ more light is required to switch the device with a $4 \mathrm{~mA}$ load current compared to the lightly loaded $1 \mathrm{~mA}$ condition. As will be seen later, the difference in optical sensitivity, which is caused by the finite gain of the amplifier, also causes the SEE response threshold to be somewhat different for different load conditions.

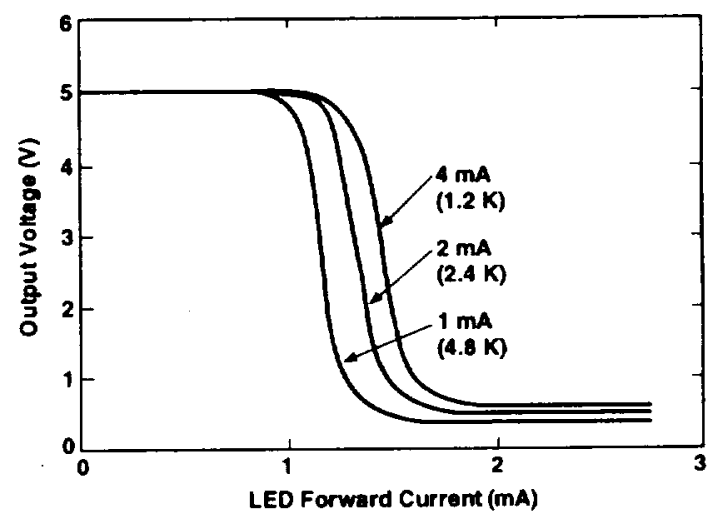

Figure 1. Output voltage vs. LED input current for three different load conditions (load resistance shown in parentheses)

\section{Experimental Results at Accelerators}

The devices were irradiated at energies of 64,95 and $195 \mathrm{MeV}$ with the Indiana University Cyclotron, and at 20 , 30 and $50 \mathrm{MeV}$ with the UC Davis cyclotron. Tests at UC Davis were done with three different load conditions, corresponding to full-scale currents of 1,2 and $4 \mathrm{~mA}$ with a $5-\mathrm{V}$ power supply. The tests at Indiana University were done with only one load condition ( $2 \mathrm{~mA}$ ). A high-speed digital oscilloscope was used to capture each individual waveform during each test run. Devices were placed in the "l" state (no current through the LED). Tests at Davis were done on devices with the lid and the LED assembly above the silicon die removed to reduce the shielding effect of those materials, which can be an important interference at low energies.

Although the output of these devices is digital for normal operation (with the LED driven well beyond the threshold conditions shown in Figure 1), tests with heavy ions or protons produce a distribution of output signal amplitudes, just as for SEE tests of digital comparators [3- 
7\}. Thus, one must set a specific triggering condition when measuring output transients. For our tests, the triggering threshold was set for $4.5 \mathrm{~V}$ (a $-0.5 \mathrm{~V}$ transient signal). Cross sections were measured by counting the number of transients that occurred for this condition, but since each individual waveform was stored it was possible to analyze the results for larger threshold triggering conditions or for other threshold criteria (such as a specific pulse width).

Tests with $195 \mathrm{MeV}$ protons energy showed no angular dependence (the lack of angular dependence at high energy was also reported by the group at GSFC [8]). A very weak angular dependence was observed at $95 \mathrm{MeV}$, and at 64 $\mathrm{MeV}$ we observed an increase in cross section of about a factor of six at the maximum angle used (87.5 degrees). The latter result is identical to the results reported by $\mathrm{LaBel}$, et al. in their initial study [1].

When the optocouplers were tested at energies below $60 \mathrm{MeV}$, the effect of angle on cross section increased dramatically compared to higher energies. Figure 2 shows the angular dependence of the cross section for the HP $6 \mathrm{~N} 134$ at three different proton energies. The cross section started to increase at much smaller angles of incidence, and the angle at which such increases first occurred was lower at lower energies.

At $30 \mathrm{MeV}$, the maximum cross section was about two orders of magnitude greater than the cross section observed at normal incidence, considerably larger than the factor of six observed at $65 \mathrm{MeV}$. At $20 \mathrm{MeV}$, interference from the package caused the cross section to fall to low values above 65 degrees, consistent with the placement of the die in the recessed package (the range of $20 \mathrm{MeV}$ protons is only about $1 / 2$ that of $30 \mathrm{MeV}$ protons). This made it impossible to determine how much the cross section would increase at more extreme angles. However, the contribution to the overall error rate is low, so that is not a serious limitation. Shielding from the package is only important at low energies, where the range of the protons is less (see Table 1).

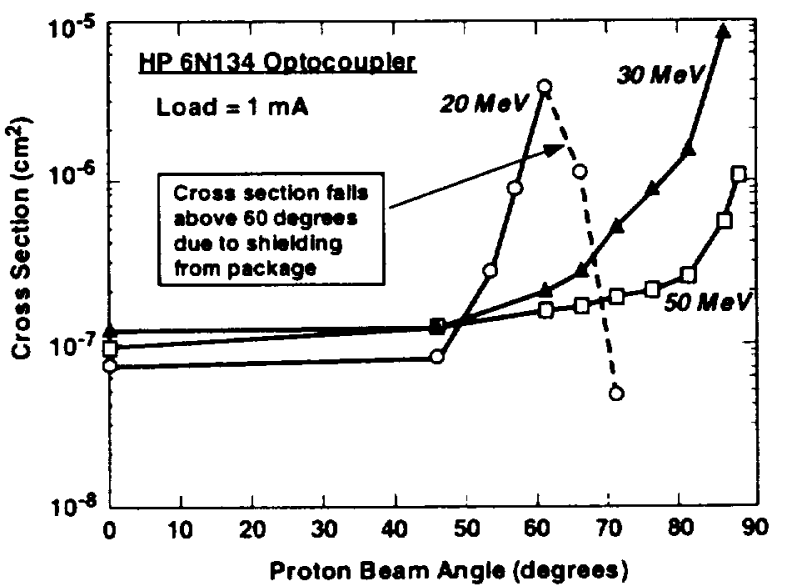

Figure 2. Effect of incident angle on cross section of the HP 6N134 optocoupler for various proton energies
The $6 \mathrm{~N} 134$ from Micropac also exhibited a strong dependence on angle of incidence. These results are shown in Figure 3; the results are very similar to the results for the $\mathrm{HP}$ version of the part, except for the $20 \mathrm{MeV}$ results. Shielding from the package occurred at larger angles for the Micropac part compared to the HP device, which is consistent with the higher position of the assembly in the package for devices from Micropac.

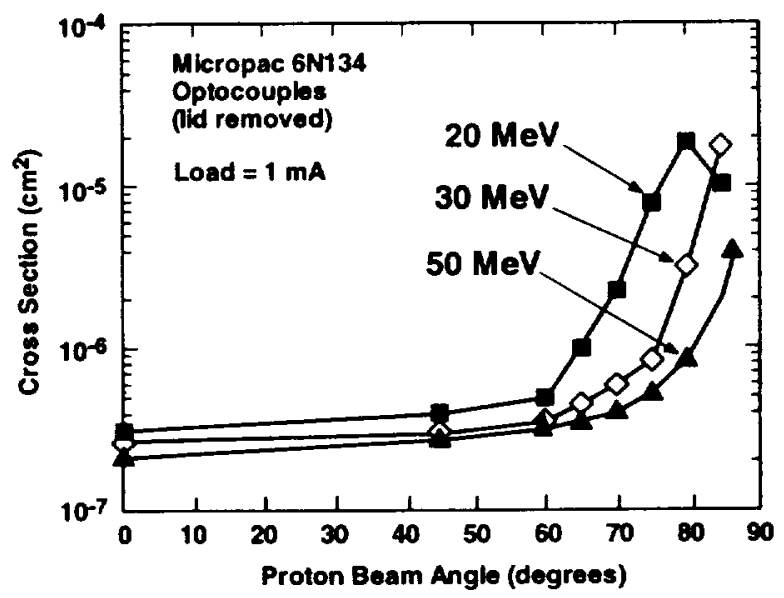

Figure 3. Effect of incident angle on cross section of the Micropac 6NI34 optocoupler for various proton energies.

The effect of different load conditions on the angular dependence of the cross section is shown in Figure $\mathbf{4}$ for the HP 6 N 134 optocoupler. When tested with a $4 \mathrm{~mA}$ load, somewhat higher angles were required in order for the angular dependence to occur. The maximum cross section was also lower for the higher load condition.

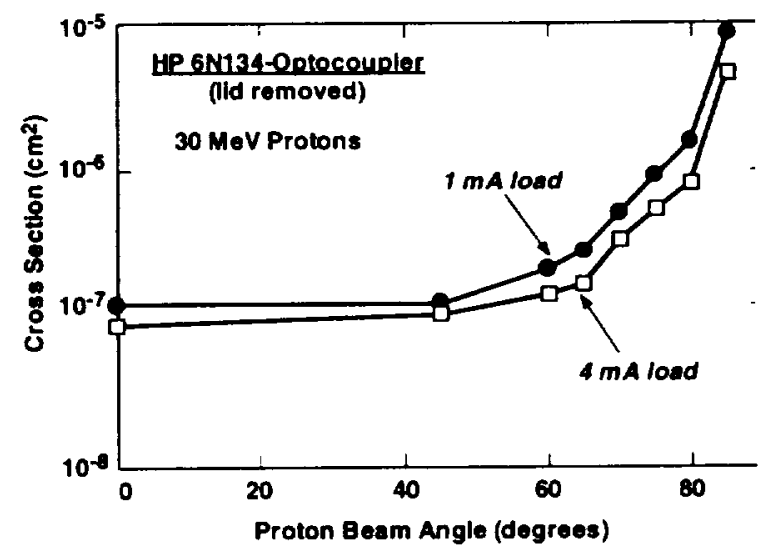

Figure 4. Effect of different load conditions on the angular dependence of the cross section of the HP 6 N 134 optocoupler with $30 \mathrm{MeV}$ protons.

\section{Discussion}

\section{A. Factors that Determine the Energy and Angular Dependence}

Two factors are involved in the proton energy dependence: the distribution of recoil energies (see references 9 and 10), and the linear energy transfer of the 
protons (direct ionization). Table I shows the LET of various proton energies, which vary by about a factor of five over the range of energies that were used in these experiments.

Table 1. LET and Range of Protons

\begin{tabular}{llcc}
$\begin{array}{c}\text { Energy } \\
\text { (MeV) }\end{array}$ & $\begin{array}{c}\text { LET } \\
(\mathrm{MeV}-\mathrm{cm} 2 / \mathrm{mg})\end{array}$ & $\begin{array}{c}\text { LET ratio } \\
\text { (compared to 65 MeV) }\end{array}$ & $\begin{array}{c}\text { Range in Si } \\
(\mu \mathrm{m})\end{array}$ \\
\hline 20 & 0.02 & 2.50 & 2,660 \\
30 & 0.015 & 1.88 & 5,220 \\
50 & 0.01 & 1.25 & 8.610 \\
65 & 0.008 & 1 & 18.000 \\
100 & 0.0064 & 0.80 & $>20,000$ \\
195 & 0.0041 & 0.51 & $>20,000$
\end{tabular}

Note that these effects are interrelated. At high energy, the mean recoil energy is higher and the LET is lower. This reduces the relative effect of the direct ionization contribution. This is consistent with the flat angular dependence observed experimentally at high energy.

At lower energies, the mean energy of the recoil products is lower and the LET is higher, increasing the relative importance of direct ionization. However, it is not possible to model the angular dependence unless the critical charge is known. The critical charge can be determined by testing devices at a particle accelerator, but it is also possible to determine critical charge using laboratory alpha particle sources, a much less costly alternative. Previous work showed that the charge collection depth in these devices is approximately $50 \mu \mathrm{m}$ [2], considerably greater than the range of alpha particles from natural nuclear decay. Energy lost in surface coatings introduces a slight error, on the order of $10-15 \%$ for these devices. Detailed corrections for overlayer thickness loss will be included in the full paper.

\section{B. Critical Charge Measurements}

A series of experiments was done with laboratory alpha particle sources to determine critical charge. Thin gold foils were interposed between the source and the device to provide several different energy values, measuring the amplitude and pulse width of the resulting transients. Three different loading conditions were used for the critical charge measurements: full-scale load conditions of 1,2 and $4 \mathrm{~mA}$. Figure 5 shows how the mean amplitude varied with alpha particle energy (more than 100 waveforms were collected for each run). For high alpha energies, most of the waveforms produce a full (saturated) output voltage, but as the energy is reduced, the mean amplitude decreases. We used the condition where the mean amplitude decreased to $1 / 2$ of full scale (in this case $2.5 \mathrm{~V}$ ) to calculate critical charge. Note, however, that the dependence of mean amplitude on energy is very steep in this region, so that the critical charge is only slightly lower if a lower mean amplitude criterion is used.

The critical charge determined in this way is shown in Figure 6. It varies between 0.055 and $0.076 \mathrm{pC}$, depending on the load condition, and would be somewhat higher with higher load conditions (these optocouplers can be used with loads up to $10 \mathrm{~mA}$ ). Note that the ratio of the critical charge is essentially the same as the ratio of the LED threshold current for the two operating conditions that was shown previously in Figure 1.

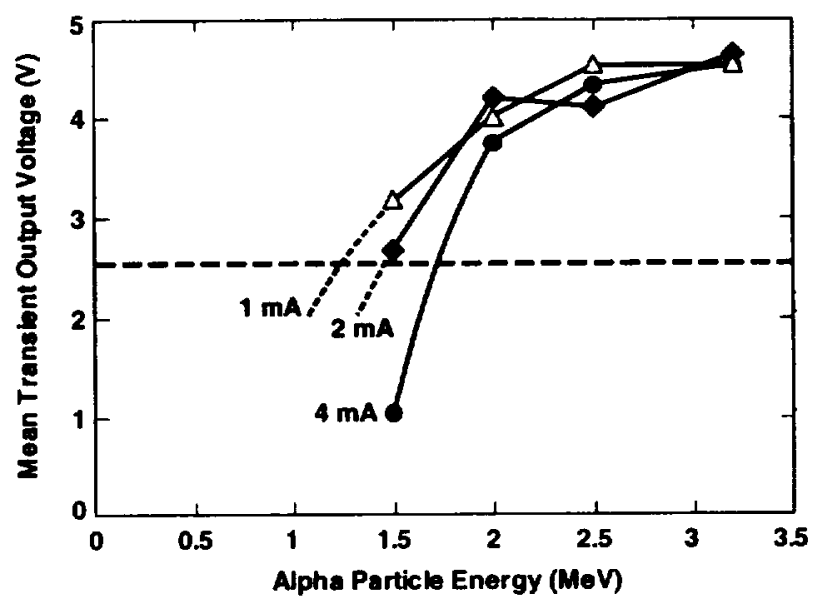

Figure 5. Determination of critical charge from laboratory alpha particle irradiations.

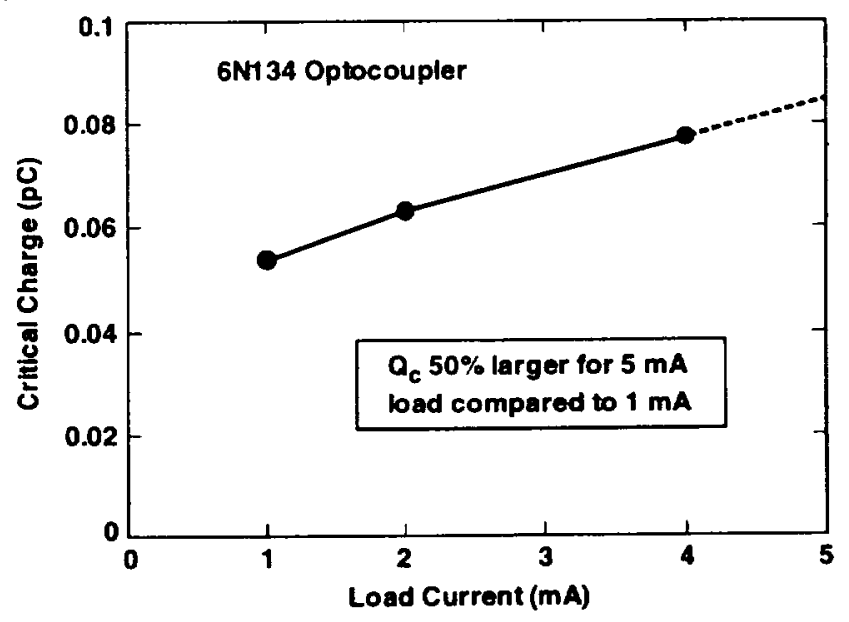

Figure 6. Critical charge for different load conditions

The critical charge measurements are in close agreement with the measured angles at which the cross section increases, assuming an inverse cosine dependence for the direct component. For example, the ratio of the secant of the angles at 1 and $4 \mathrm{~mA}$ load conditions of Figure 3 is 1.30 , nearly the same ratio as the critical charge for the two load conditions in Figure 6 (1.34).

Only certain types of optocouplers are affected by proton upset. It is possible to determine whether direct ionization effects are important by combining laboratory measurements of critical charge with the physical 
measurement of the photodiode. The maximum charge at extreme angles is simply the product of the LET of protons at various energies and the diameter of the photodiode, as supported by the data at different energies and load conditions.

\section{System Implications}

The effect of the increase in angular dependence of cross section depends on the proton energy spectrum, as well as the angular dependence. In many cases, far more shielding is present than assumed in the initial specifications because of the presence of circuit boards and other physical assemblies on the spacecraft. Thus, the actual proton spectrum may have a much higher mean energy than stated in the initial requirements (which usually assume a spherical shield of nominal thickness). This will reduce the significance of the direct component in many instances.

For example, consider a polar orbit $\left(98^{\circ}\right)$ at $705 \mathrm{~km}$ (used by several systems that monitor climate conditions on the Earth). The calculated number of transients from an HP $6 \mathrm{~N} 134$ optocoupler is 10.5 times higher with a 100 mil spherical shield when the direct ionization effect is taken into account compared to the number from indirect proton reactions alone. This difference is reduced to a factor of four for a shielding thickness of 400 mils of aluminum. The key points are that shielding has a large influence on the net upset rate, and that the direct ionization component is expected to be more important for small spacecraft with less shielding.

\section{Conclusions}

This paper has shown that direct ionization causes a very large increase in the cross section for single-event transients in optocouplers. The magnitude and the angle at which the direct ionization component affects the response increases as the proton energy decreases, which is consistent with the energy dependence of LET. The maximum increase in cross section that was observed is about 100 , but somewhat larger factors may occur at lower energies. Direct ionization was observed at incident angles as low as $45^{\circ}$, far lower than the earlier work at high energies where it was only significant for very large angles of incidence.

A technique for measuring critical charge with laboratory alpha particle sources was demonstrated that can be used to determine whether proton ionization is likely to be important for specific devices. This is potentially important as a hardness assurance tool as well as in corroborating the direct ionization mechanism.

\section{References}

I. K. LaBel, et al., "Proton-Induced Transients in Optocouplers: InFlight Anomalies, Ground Irradiation Test, Mitigation and Implications," IEEE Trans. Nucl. Sci., 44, 1895 (1997).

2. A. H. Johnston, et al., "Single-Event Upset Effects in Optocouplers," IEEE Trans. Nucl. Sci., 45, 2867 (1998).

3. R. Koga, et al., "Observation of Single-Event Upsets in Analog Microcircuits." IEEE Trans. Nucl. Sci.. 4Q, 1838 (1993).

4. R. Ecoffet, et al., "Observation of Heavy lon Transients in Linear Circuits," 1994 IEEE Radiation Effects Data Workshop. IEEE 94TH08641, p. 72.

5. D. K. Nichols, et al., "Heavy Ion and Proton Induced Single Event Transients in Comparators." IEEE Trans. Nucl. Sci., 43, 2960 (1996).

6. T. L. Turflinger, "Single Event Effects in Analog and Mixed-Signal Integrated Circuits," IEEE Trans. Nucl. Sci., 43, 594 (1996).

7. R. Koga, et al., "Single Event Upset Sensitivity Dependence of Linear Integrated Circuits on Bias Conditions." IEEE Trans. Nucl. Sci., 44, 2235 (1997).

8. M. V. O'Bryan, et al., "Single Event Effects and Radiation Damage Results for Candidate Spacecraft Electronics," 1998 IEEE Radiation Effects Data Workshop. IEEE 98TH08385, p. 39.

9. G. E. Farrell and P. J. McNulty, "Microdosimetric Aspects of ProtonInduced Nuclear Reactions in Thin Layers of Silicon," IEEE Trans. Nucl. Sci., 29, 2012 (1982).

10. P. J. McNulty, "Single-Event Effects Experienced by Astronauts and Microelectronic Circuits Flown in Space," IEEE Trans. Nucl. Sci., 43. 475 (1996). 